\title{
Temperature and Humidity Monitoring System for Embedded Computer
}

\author{
Zhu Lei1, Wang Yongjie1, Li Siwei2, Liu Heng1 \\ 1No. 63981 Unit of PLA, Wuhan, China \\ 2Experimental Instrument Plant, Academy of Military Medical Sciences, \\ Beijing, China \\ 1Email: zhulei1120@126.com \\ 2Email: lsw2005010608@163.com
}

\begin{abstract}
Temperature and humidity is the key feature in the process of equipment airproof storage, and its monitoring precise influences the effect of the airproofing of equipment, consequently, influences its battle performance. This paper introduces the design and realization of temperature and humidity monitoring system for airproof storage. Testing and controlling scheme based on PC104 bus is provided as well as the constitution of hardware and the design of software. The problems of maintenance and management in the process of equipment storage can be pertinently resolved. Results show that the temperature and humidity intelligent monitoring system runs stably, and has good human-machine interactivity as well as high general and extensibility.
\end{abstract}

Keywords: temperature and humidity; monitoring system; PC104 bus; dehumidifier

\section{Introduction}

The temperature and humidity should be controlled in the process of equipment airproof storage. If temperature and humidity cannot meet the criterion, there may be corrosion and mildew on machinery and electron system, which will cause economy loss and influence the fighting capacity. The traditional storage mode is to put the equipment in the air, and administrator detects the temperature and humidity regularly. When temperature and humidity cannot meet the criterion, the administrator will open the door and window, or turn on the air conditioning. For the warehouses equipped dehumidifier, the administrator will use dehumidifier to control temperature and humidity. Usually, the working time is determined by experience, which will always cause wasting. The detection precision is low. Obviously, the traditional storage mode is unscientific and uneconomic for storage of equipment needing exact environment requirement [1]. To improve the condition, large removable hermetic wrapper is used in the process of equipment airproof storage. Neighborhood wrappers are connected with pipes to build a airproof storage 
environment. Dehumidifier or other dehumidification equipments for temperature and humidity automatic regulation is used to make sure equipment airproof store in dependable environment, to avoid adverse effect caused by environment stress[2]. The composition of equipment airproof storage is showed in Fig.1. It can be seen in Fig.1 that airproof storage system is composed with temperature and humidity monitoring system, dehumidifier, wrapper and blowpipe, and temperature and humidity monitoring system is the most important part of whole system.

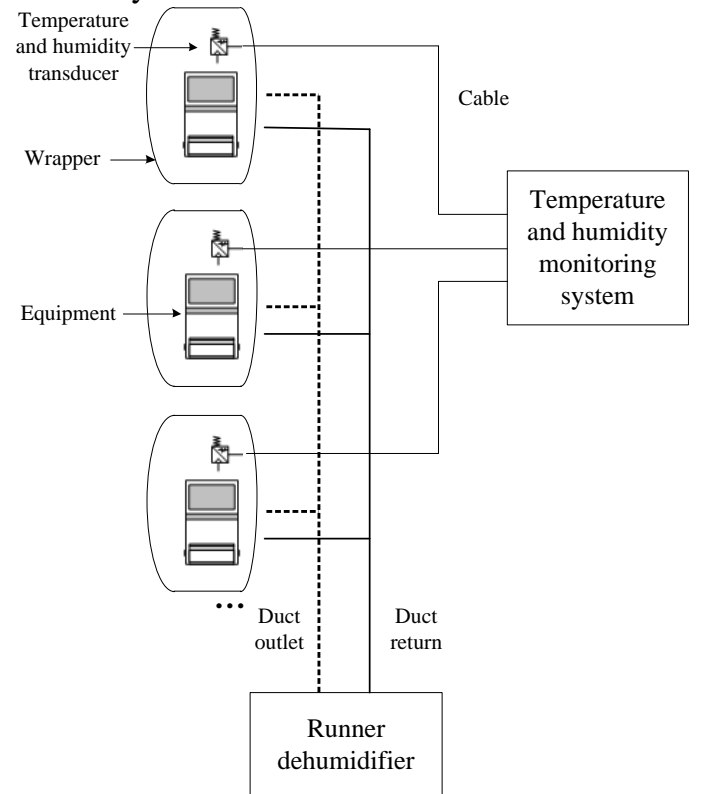

Figure 1. Composition of Equipment Airproof Storage.

So far, there are some common temperature and humidity monitor modes, such as man-made measurement mode, dispersion instrument control mode, centered computer monitoring system mode. The monitoring system with industrial control main board core belonging to entered computer monitoring system mode is used in the paper, and it is based on temperature and humidity transducer, and the core [3].

\section{System requirement analysis and overall design}

System Requirement Analysis. Every wrapper in airproof storage environment represents a humidity detection point needing a temperature and humidity transducer. For the temperature head of wrappers is small, only one temperature detection point is needed. Considering the capacity of dehumidifier, 15 wrappers compose an airproof storage system. So the monitoring system needs 16 collection channels, one for temperature collection, 15 for humidity collection. For temperature and humidity change slow, the sampling frequency is larger than $100 \mathrm{~Hz}$. The temperature and humidity should be displayed real-time in all 
detection points and the temperature and humidity data should be stored. If abnormal occurrence appears, signal should be given to administrator for mastering the temperature and humidity condition. Dehumidifier is controlled by system to monitor temperature and humidity automatically.

Overall Design.Temperature and humidity intelligence monitoring system is composed with PC104 industrial control main board, PC104 data collection card, controlling card, temperature and humidity transducer, LCD screen, power supply module and SD card[4].

The basic working process is as follow: after regulation, electricity signal of temperature and humidity transducer is transferred to data collection card; the collection temperature and humidity is transferred to PC104 embedded industrial control main board through PC104 bus; the collection data is processed by embedded system and is compared with threshold; the control signal is generated by GPIO card to control dehumidifier using controlling card. Meanwhile, huge temperature and humidity data is collected by SD card, and the real-time temperature and humidity data is showed by LCD screen. System principle is showed in Fig.2.

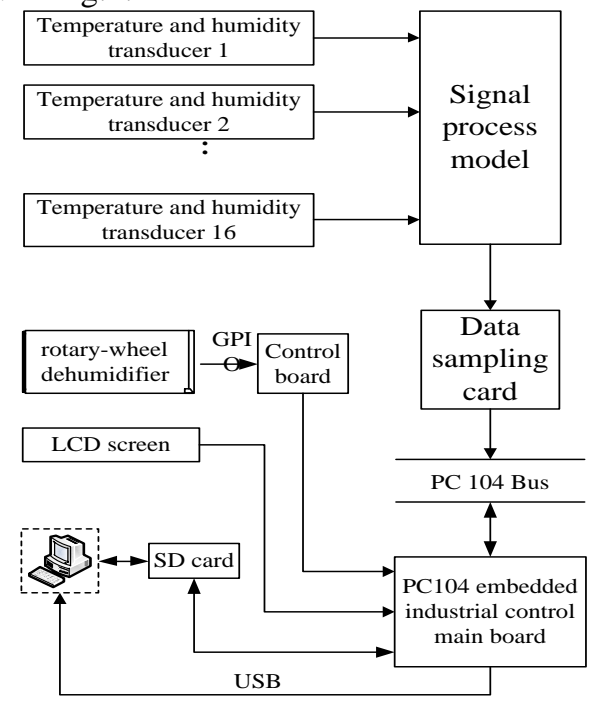

Figure 2. Principle of Temperature and Humidity Monitoring System

\section{System Hardware Composition}

PC104 Embedded Industrial Control Main Board.EPC-8600 industrial control main board is used for host computer in monitoring system. EPC-8600 is PC104 industrial control main board based on PXA270 transducer. It has advantages of abundance resource, complete interface, low power consumption, and high reliability [5]. Its structure has excellent antivibration. Using PC104 industrial control main board as host computer, hardware circuit design is easy, and the 
system data can be stored into CF card to avoid data loss, which can provide sufficient data for administrator.

Signal Regulation Modular.The function of signal regulation modular is to transform input signal of transducer into collection signal. To decrease the signal transmission loss on the line, the system temperature and humidity transducer is current export mode. $250 \Omega$ resistance is used to transform $4 \sim 20 \mathrm{~mA}$ current signal into $1 \sim 5 \mathrm{~V}$ voltage signal for collection.

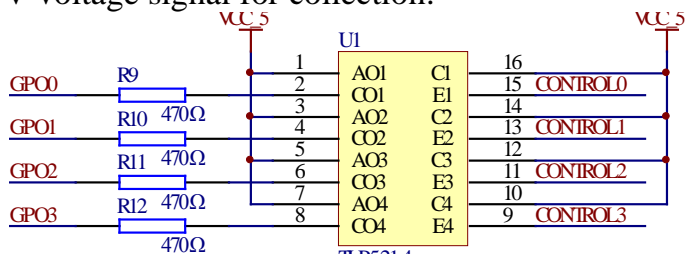

Figure 3. Opticalcoupler Isolation Principle

Data Collection Card.PCM-8208BT card is chosen as data collection card that is intelligence high speed analogue input card based on PC104 bus. It has 16 input channels that can be equipped to finite difference input channel and it also has 5 grades adjustable gain amplifier with input range from $\pm 0.625 \mathrm{~V}$ to $\pm 10 \mathrm{~V}$. The sampling precision is 12 bit, and sampling frequency is $48 \mathrm{kHz}$. Input port has overtension protection.

Controlling Card.PC104 embedded industrial control main board integrates eight general purpose $\mathrm{I} / \mathrm{O}$. to decrease cost price and to increase the system practicality, controlling card is designed for controlled object. The size of controlling card is $96 \mathrm{~mm} \times 90 \mathrm{~mm}$, being as same as that of standard PC104 modular. the cardinal plate and collection card is connected by copper column to decrease space usage and to strengthen system structure.

Four channels isolation output are designed by controlling card to control dehumidifier or other dehumidification equipments. Two stage isolation methods are chosen: the first one use TLP521-4 photoisolator for opticalcoupler isolation, and the second one use G5LA-14 5VDC for relay isolation.

As the output voltage of embedded industrial control main board is low level, photoisolator breakovers. The output high level controlled signal is used for input signal of next relay. One $470 \Omega$ resistance connects the 4 channels GPO of PC104 cardinal plate and TLP521-4 photoisolator. The resistance is used to limit the current to protect the photoisolator. It is showed in Fig.3.

For output of one channel, GPO0 is set in low level, meanwhile before CONTROL0 is in high level, then triode Q1 breakovers and relay RE1 attracts. Then, dehumidifier works, and light L1 turns on. It is showed in Fig.4. R1 resistance is used to limit the current to protect the base. Diode D1 is used to suppress opposite voltage of relay coil to protect triode Q1. 


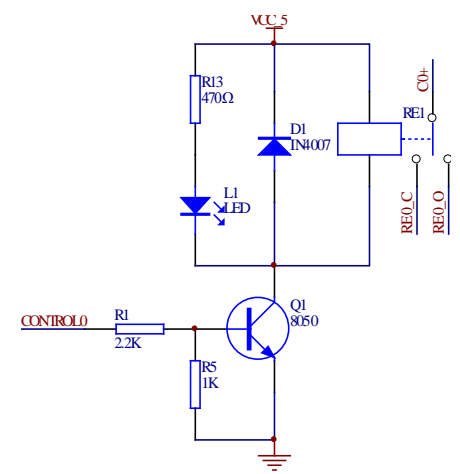

Figure 4. Relay Isolation Output Principle

Other Functional Modules.LQ104V1DG52 is used as system LCD screen. Its size is 10.4 inch, and its resolution is $640 \times 480$.

CCFT(Cold Cathode Fluorescent Tube) is used in the LCD screen, with voltage modular providing $12 \mathrm{~V} \sim 26 \mathrm{~V}$. The voltage of temperature and humidity transducer is $24 \mathrm{~V}$; voltage of embedded industrial control main board is $5 \mathrm{~V}$; voltage of controlling is $5 \mathrm{~V}$. So, dual channel switching power supply (24V and $5 \mathrm{~V}$ ) is chosen as system power supply.

\section{System Software Design}

The operating system is Windows CE in the paper. Windows CE system is embedded operating system developed by Microsoft Corporation, and is widely used in many fields, such as regulation, communication and multimedia [6]. Windows CE is information equipment platform based on Win32 API, with character of modularization, processor independence and structure [7]. Meanwhile, visualization instrument Embedded Visual $\mathrm{C}^{++}$is developed for software by Microsoft Corporation. Software design flow diagram of monitoring system is showed in Fig.5. 


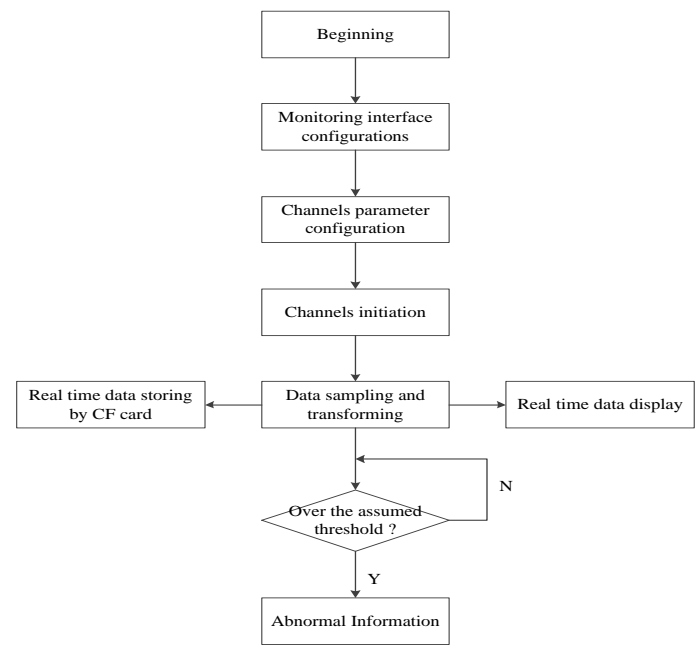

Figure 5. Software Design Flow Diagram

Two interface configurations are optional in the monitoring system; they are nixie light and text. Nixie light is used to display channel voltage or system time, and text is used to display character information. The system is equipped after configuration, and the equipped parameters include $\mathrm{AD}$ channel selection, channel name installation, standardization, warning boundary, base address of PC104 card, IRQ channel, measuring range and sampling frequency. Each channel is initialized after parameters equipment, and the data is sampled. Data is displayed by LCD screen and is stored by CF card for administrator analyzing the temperature and humidity data.

The system warning humidity threshold is 55 . That is to say, when relative humidity is over $55 \%$, the system warns. When relative humidity of anyone wrapper is over $55 \%$, dehumidifier is opened; when humidity of all wrappers is lower than $45 \%$, dehumidifier is closed.

\section{Conclusion}

The monitoring system is used in one warehouse. It indicates that the monitoring system has character of small volume, light weight, automation, intelligentification. The system can control the dehumidifier to regulate temperature and humidity of airproof storage automatically. The system runs responsibly, and can protect the performance of battle equipments efficiently, gaining great economic benefit.

The system not only can be used for temperature and humidity of airproof storage, but also can be used in granary, warehouse and exhibition hall [8] to provide technique for temperature and humidity monitoring. Using warning function of voice and light to remind workman for examination, and using control function for regulation of temperature and humidity, the system gains unattended operation. 


\section{Reference}

[1] WU Fuxin, FENG Tongbo, WU Canwei: Equipment Enviromental Engineering. Vol. 6(2009), p.74-76.

[2] HU Liangyong, XU Zongchang, LEI Yusheng:World Standardization \& Quality Management. Vol. 5 (2008),p.45-48.

[3] WANG Yila: Chang'an University master degree thesis, 2009.

[4] ZHANG Ni, DUAN Wenqiang, SHAO Tingting: Journal of Yanan University (Natural Science Edition). Vol.29(2010) ,p.50-52.

[5] WU Min: Public Communication of Science \& Technology. Vol. 13 (2010) ,p.186.

[6] ZHOU Yulin, Ning Yang, Lu Guiqiang, in: Application Development and inner core production of Windows CE.net, edited by FU Lin, Feature of Embedded Operating System ,chapter,6,Publishing House of Electronics Industry(2005).

[7] LI Wenxin, WANG Guanglong, CHEN Jianhui: Design Engineering. Vol.18 (2010), p.139-142.

[8] YANG Yaning, WU Yinghui: Journal of Dalian Nationalities University. 2010, Vol.12 (3), p. 220-223. 